Voix et Images

volxetimages

\title{
Trois siècles d'affiches françaises : image et sémiologie
}

\section{Raymond Montpetit}

Volume 2, numéro 2, décembre 1976

Paul Chamberland

URI : https://id.erudit.org/iderudit/200063ar

DOI : https://doi.org/10.7202/200063ar

Aller au sommaire du numéro

Éditeur(s)

Les Presses de l'Université du Québec

ISSN

0318-9201 (imprimé)

1705-933X (numérique)

Découvrir la revue

Citer cet article

Montpetit, R. (1976). Trois siècles d'affiches françaises : image et sémiologie.

Voix et Images, 2(2), 290-293. https://doi.org/10.7202/200063ar d'utilisation que vous pouvez consulter en ligne.

https://apropos.erudit.org/fr/usagers/politique-dutilisation/ 
Trois siècles d'affiches françaises: image et sémiologie

Le Musée des beaux-arts de Montréal nous présentait à l'automne "Trois siècles d'affiches françaises" une exposition conçue et réalisée par 
le Musée des arts décoratifs de Paris et prêtée à plusieurs musées canadiens (Montréal, Toronto, Victoria, Winnipeg, Rimouski). Même si la disposition et l'accrochage de cette exposition à Montréal étaient fort mauvais et improvisés (des affiches étaient étalées pêle-mêle dans l'entrée, sans identification aucune), il n'en reste pas moins que les affiches montrées étaient intéressantes et permettaient de disposer d'une rétrospective concise illustrant l'évolution de la nature et du style de l'affiche française. Nous voudrions profiter de cette occasion pour indiquer quelques pistes de réflexion au sujet des images et de leur fonctionnement muséologique.

L'affiche opère à l'intérieur de la culture une démystification de l'image, elle marque en quelque sorte la fin du caractère magique (latent) de sa production: grâce à l'invention de la lithographie (1798) et ensuite de la photographie, les images se multiplient, perdent leur rareté et deviennent un phénomène courant et commun (non précieux), ce qui a pour effet de briser le lien traditionnel entre art et image. La majorité des images produites et consommées aux $\mathrm{XIX}^{\mathrm{e}}$ et $\mathrm{xx}$ e siècles, ne sont pas considérées comme "artistiques", ces secteurs étant désormais nettement distingués, et il faut relier cette scission à cette autre brisure qui donnera naissance à l'art pictural abstrait ; en effet, à partir du moment où il y a image sans art, il peut aussi y avoir de l'art sans perception d'image, production picturale non imagée qui réponde à ces images non artistiques. Dans cette perspective, l'un des stimuli de l'art abstrait apparaît être une réaction élitiste face à la popularisation des images dans le but d'affirmer une pureté plastique qui ira contre ces images toujours au service d'autre chose et s'y référant.

L'affiche imagée remplit donc une fonction et l'art cherchera à se définir en dehors d'elle... et pourtant voilà bien les affiches au musée, comme par un certain retour des choses; voyons ce retournement de plus près.

Les images cumulent, comme les énoncés linguistiques, plusieurs fonctions qui peuvent d'ailleurs servir de principe classificateur; les six fonctions décrites par Roman Jakobson et se rattachant à chacun des éléments de la communication linguistique (voir à ce sujet «Linguistique et poétique " in Essais de linguistique générale) se transposent aux affiches, et l'on peut alors constater que celles-ci misent principalement sur la fonction conative liée au destinataire, sur la fonction phatique qui voit à attirer l'attention et à établir le contact, et, sur la fonction référentielle qui nous décrit le produit à promouvoir. Mais, lorsque l'on regarde, au musée, ces affiches du temps passé, il se produit bien quelque chose comme un détournement fonctionnel, une mutation dans les mécanismes de l'affiche.

En premier lieu, ces affiches ne nous sont pas destinées, ou plus précisément, nous ne sommes pas leurs destinataires, puisque pour nous il est déjà trop tard, (trop tard pour aller entendre Aristide Bruant ou voir La 
Goulue) et qu'il n'est même pas certain que nous soyons en mesure de saisir les sens qu'elles produisent; en second lieu les contextes vers lesquels elles pointent référentiellement, sont le plus souvent disparus et ne correspondent plus à rien dans la structure de notre comportement de consommation. Impossible donc de lire l'affiche comme si elle n'était qu'affichée: elle est ici exposée au musée, ce qui nous force à opérer une lecture qui va à l'encontre de la hiérarchie des fonctions que l'affiche elle-même établissait, en abolissant référence et interpellation persuasive au profit d'une contemplation esthétique non utilitaire, qui a vite fait de métamorphoser en "art " tout objet étiqueté et exposé dans un cadre officiel approprié. Une affiche particulière, prenant alors place dans une série, devient lisible en fonction de toutes les autres, manifestant ainsi ses spécificités stylistiques et exprimant son auteur-concepteur, bref fonctionnant d'une tout autre façon et dans un tout autre réseau que lorsqu'elle était sur les murs de la ville au service de son référent à vendre aux passants.

Cette inscription de l'affiche dans son propre réseau historique rend manifeste ce que l'on peut appeler son «intertextualité" ou encore mieux son «interconicité, c'est-à-dire les relations multiples qui, par rapports associatifs comme disait Saussure, relient une image à plusieurs autres à l'intérieur de la mémoire culturelle. Une affiche imagée, comme un terme, est "le centre d'une constellation", et il nous semble que la série associative qu'elle engendre et qui l'englobe suit un tracé similaire à celui que Saussure décrit pour un terme donné de la langue. Une image pourrait donc être considérée comme entretenarit avec plusieurs autres «in absentia", des rapports associatifs ou paradigmatiques, que l'exposition au musée a l'avantage de rendre plus explicites.

Saussure précise dans son Cours de linguistique générale (Deuxième partie, chapitre $V$ ), quatre axes associatifs principaux qui relient un terme à d'autres termes de la langue, soit:

1. l'axe par le radical lorsque deux termes ont un même radical,

2. l'axe de la synonymie, deux termes s'appelant parce qu'ils ont à peu près le même signifié,

3. l'axe par le suffixe, deux termes ayant comme élément commun leur suffixe,

4. l'axe par l'image acoustique, lorsque deux termes s'entendent de façon similaire jouant sur les mêmes sons.

Ces quatre types de relations paradigmatiques peuvent se transposer utilement au domaine des images, et permettre d'élucider la nature des associations qui relient telle image à une autre: nous considérerions alors qu'une image entretient avec une autre un rapport paradigmatique; (1) par son radical, si une partie de l'image, tel décor, tel personnage, se retrouve dans l'autre; (2) par synonymie, si la signification globale des deux images 
est la même, par exemple l'amour, bien que les formes visuelles changent; (3) par suffixe, lorsque deux images ont été obtenues à partir d'un même principe de composition spatiale, par exemple deux triptyques, ou deux images présentant une opposition d'obliques, etc.; et (4) par l'image acoustique, devenant ici la gamme des couleurs lorsque deux images présentent les mêmes pigments colorés.

L'histoire de l'affiche est la manifestation de ces rapports qui créent entre les affiches des liaisons fort différentes de celles qu'elles avaient pour but initial d'instaurer entre un produit et un client possible.

Au musée, les affiches s'indiquent l'une l'autre, en nous rappelant la constellation paradigmatique dont elles font partie: il nous semble qu'il y a là l'illustration de la pertinence de certains concepts de la linguistique structurale pour la compréhension du fonctionnement des images.

Raymond Montpetit 